\title{
Characterization of insect galls, gall makers, and associated fauna of Platô Bacaba (Porto de Trombetas, Pará, Brazil)
}

\author{
Valéria Cid Maia ${ }^{1,2}$ \\ ${ }^{1}$ Departamento de Entomologia, Museu Nacional, Quinta da Boa Vista, São Cristóvão, \\ CEP 20940-040, Rio de Janeiro, RJ, Brazil \\ ${ }^{2}$ Corresponding author: Valéria Cid Maia,e-mail: maiavcid@acd.ufrj.br
}

MAIA, V.C. Characterization of insect galls, gall makers, and associated fauna of Platô Bacaba (Porto de Trombetas, Pará, Brazil). Biota Neotrop. 11(4): http://www.biotaneotropica.org.br/v11n4/en/ abstract?article+bn00511042011

\begin{abstract}
Seventy six morphotypes of insect galls were found on 38 plant species and one subspecies distributed among 27 genera and 22 families in Platô Bacaba (Porto de Trombetas, Pará, Brazil). The majority of these galls (about $80 \%$ ) occurred on leaves and $20 \%$ on stems. Burseraceae was the plant family with the greatest number of gall morphotypes $(\mathrm{N}=23)$, followed by Fabaceae $(\mathrm{N}=11)$ and Melastomataceae $(\mathrm{N}=6)$. Protium Burm. $\mathrm{f}$. $(\mathrm{N}=17)$, Inga Miller $(\mathrm{N}=8)$, and Tetragastris Gaertn $(\mathrm{N}=6)$ were the plant genera, and Protium sagotianum Marchand $(\mathrm{N}=7)$, Tetragastris panamensis $(\mathrm{N}=6)$, and Miconia stenostachya DC. $(\mathrm{N}=5)$ were the plant species that supported the highest diversity of galls. The galling inducers belong to Diptera (Cecidomyiidae) and Lepidoptera orders. Galls of Coleoptera, Hymenoptera, Hemiptera and Thysanoptera were not found. This study adds evidences that Diptera (Cecidomyiidae) are the most frequent galling insects in different zoogeographical regions.
\end{abstract}

Keywords: Amazonian Forest, Cecidomyiidae, Diptera, diversity, insect galls, gallers.

MAIA, V.C. Caracterização das galhas de insetos, galhadores e fauna associada do Platô Bacaba (Porto de Trombetas, Pará, Brasil). Biota Neotrop. 11(4): http://www.biotaneotropica.org.br/v11n4/pt/ abstract?article+bn00511042011

Resumo: Setenta e seis morfotipos de galhas de insetos foram encontrados em 38 espécies de planta e uma subespécie distribuídos em 27 gêneros e 22 famílias vegetais no Platô Bacaba (Porto de Trombetas, Pará, Brasil). A maioria dessas galhas (cerca de $80 \%$ ) ocorreu em folhas e $20 \%$ em caules. Burseraceae apresentou maior número de morfotipos de galhas $(\mathrm{N}=23$, seguida por Fabaceae $(\mathrm{N}=11)$ e Melastomataceae $(\mathrm{N}=6)$. Protium Burm. $\mathrm{f}$. ( $\mathrm{N}=17)$, Inga Miller $(\mathrm{N}=8)$, e Tetragastris Gaertn $(\mathrm{N}=6)$ foram os gêneros botânicos e Protium sagotianum Marchand $(\mathrm{N}=7)$, Tetragastris panamensis $(\mathrm{N}=6)$ e Miconia stenostachya $\mathrm{DC}$. $(\mathrm{N}=5)$ foram as espécies vegetais que suportaram maior diversidade de galhas. Os indutores de galha pertencem às ordens Diptera (Cecidomyiidae) e Lepidoptera. Galhas de Coleoptera, Hymenoptera, Hemiptera e Thysanoptera não foram encontradas. O trabalho adiciona mais evidências que Diptera (Cecidomyiidae) são os principais insetos indutores de galhas em diferentes regiões zoogeográficas.

Palavras-chave: Floresta Amazônica, Cecidomyiidae, Diptera, diversidade, galhas de insetos, galhadores. 


\section{Introduction}

Little is known about galling insects of Amazonian Forest. In spite of being a very rich ecosystem, with more than 1,000 gall morphotypes (Oda 2006, Julião 2007), an inexpressive number of galling species has been identified (only 22 species of Cecidomyiidae, Diptera). Taxonomical studies of this fauna were developed by Rübsaamen (1895, 1905, 1916a,b), Felt (1908, 1911a,b, 1915, 1921) and Kieffer $(1895,1913)$. These authors are responsible for about $80 \%$ of the galling species records. Other records were made by Molliard (1903), Silvestri (1901), Möhn (1960, 1962), Gagné (1969, 1977, 1994), Maia \& Vasquez (2006), Maia \& Fernandes (2006), and Fernandes \& Maia (2010).

The Amazonian Forest has an area of about 7 millions of $\mathrm{km}^{2}$ and spreads over Brazil, Bolivia, Colombia, Ecuador, Guiana, French Guiana, Peru, Suriname, and Venezuela. Approximately, $60 \%$ of this extension is situated in Brazil, where it spreads over 7 states: Amazonas, Amapá, Mato Grosso, Maranhão, Pará, Rondônia, and Tocantins. This survey was developed in an area of dense forest in Platô Bacaca (Pará, Porto de Trombetas, FLONA Saracá-Taquera $\left(1^{\circ} 20^{\prime}-1^{\circ} 55^{\prime} \mathrm{S}\right.$ and $\left.56^{\circ} 00^{\prime}-57^{\circ} 15^{\prime} \mathrm{W}\right)$, which has never been investigated before and is part of an environmental monitoring program.

The main objective of this work is to survey and characterize the insect galls of the Platô Bacaba. In addition, information on biodiversity of insect galls in different localities is provided. Scattered data were compiled to give an overview of it and to allow comparisons among the areas.

\section{Material and Methods}

Insect galls were collected in Platô Bacaba (Porto de Trombetas, Pará, Brazil) during three consecutive days for 6 hours in April, 2010, totalizing 18 hours of field work. Four transects (each one with four $200 \mathrm{~m}$ lines in intervals of 25, 100, 250 and $500 \mathrm{~m}$ ) were settled in the studied area, one on the forest edge, two in the mountainside areas and the other at the top of Platô. The vegetation was examined along each line in search of galling insects during 4 hours. All plant organs were investigated, except for subterranean roots.
Samples of each host plants were pressed for preservation and they were later identified by Dr. Gracialda Costa Ferreira (Universidade Federal Rural da Amazônia, Brazil).The dried specimens were incorporated into the herbarium of the Instituto de Ciências Agrárias (Pará, Brazil).

Samples of each gall morphotypes were photographed, collected and transported individually in labeled plastic bags.

Larvae and pupa of immature insects were obtained from the dissection of each morphotype of gall under a stereoscopic microscope. This procedure also enabled the determination of the gall dwellers' habits. The pupal exuviae and adults were obtained from rearing, by keeping samples of each kind of gall individually in covered plastic pots with damp cotton at the bottom. These pots were examined daily for adults' emergence. The galls were kept in these rearing pots until their deterioration.

All insects were preserved in $70 \%$ alcohol. The gall midges (larvae, pupae, pupal exuviae and adults) were later mounted on microscope slides following the methodology of Gagné (1989). The Cecidomyiidae genera were identified based on the keys of Gagné (1994). The insects were incorporated in the entomological collection of Museu Nacional (MNRJ).

\section{Results}

Seventy six morphotypes of insect galls were found on 38 plant species and one subspecies, distributed among 22 families in Platô Bacaba (Porto de Trombetas, Pará, Brazil). The medium number of gall morphotypes per plant species was 1.95 (Table 1). In spite of adopting different methodologies, several authors have found similar data in other areas of Amazonian Forest, such as Central Amazonia $(\bar{X}=2.1)$ and Tapajós ( $\bar{X}=2.0)$, as well as in other ecosystems, such as "restinga" (coastal shrubs) in Bertioga (São Paulo state) $(\bar{X}=1.9)$, Maricá and Carapebus (Rio de Janeiro state) $(\bar{X}=2.1)$, and "cerrado" (Brazilian savanna) in Serra de São José (Minas Gerais state) $(\overline{\mathrm{X}}=1.9)$ (Table 2).

Burseraceae were the richest plant family in number of gall morphotypes $(\mathrm{N}=23)$, following by Fabaceae $(\mathrm{N}=11)$, and Melastomataceae $(\mathrm{N}=6)$. Protium Burm. f. $(\mathrm{N}=17)$, Inga Miller

Table 1. Richness of insect galls on plant families and species in Platô Bacaba (Porto de Trombetas, Pará, Brazil).

Tabela 1. Riqueza de galhas de insetos nas famílias e espécies de planta em Platô Bacaba (Porto de Trombetas, Pará, Brasil).

\begin{tabular}{|c|c|c|}
\hline Plant family & Plant species & $\begin{array}{c}\text { Number of gall } \\
\text { morphotypes }\end{array}$ \\
\hline Achariaceae & Lindackeria atifolia Benth. & 1 \\
\hline \multirow[t]{2}{*}{ Bignoniaceae } & Arrabidaea sp. & 3 \\
\hline & Memora magnifica (Mart. ex DC.) Bureau & 1 \\
\hline \multirow[t]{2}{*}{ Boraginaceae } & Cordia scabrifolia A.DC. & 1 \\
\hline & Cordia sp. & 1 \\
\hline \multirow[t]{6}{*}{ Burseraceae } & Protium giganteum Engl. & 1 \\
\hline & Protium cf. giganteum Engl. var. giganteum & 4 \\
\hline & Protium paniculatum Engl. & 1 \\
\hline & Protium sagotianum Marchand & 7 \\
\hline & Protium spruceanum (Benth.) Engl. & 4 \\
\hline & Tetragastris panamensis (Engl.) Kuntze & 6 \\
\hline Cecropiaceae & Pourouma guianensis Aubl. & 3 \\
\hline \multirow[t]{2}{*}{ Chrysobalanaceae } & Licania blackii Prance & 1 \\
\hline & Licania longistyla (Hook. f.) Fritsch & 1 \\
\hline
\end{tabular}


$\frac{\text { Table 1. Continued... }}{\text { Plant family }}$

Plant species

Number of gall morphotypes

\begin{tabular}{|c|c|c|}
\hline Euphorbiaceae & Alaeophora sp. & 1 \\
\hline \multirow[t]{5}{*}{ Fabaceae } & Inga cayennensis Sagot ex Benth. & 1 \\
\hline & Inga coriacea var. leptopus (Benth.) J.F.Macbr & 1 \\
\hline & Inga rubiginosa (Rich.) DC. & 3 \\
\hline & Inga umbellifera (Vahl) Steud. & 3 \\
\hline & Zygia racemosa (Ducke) Barneby \& J.W.Grimes & 3 \\
\hline Lacistemataceae & Lacistema polystachyum Schnizl. & 1 \\
\hline \multirow[t]{2}{*}{ Lauraceae } & Aniba burchellii Kosterm. & 1 \\
\hline & Aniba sp. & 1 \\
\hline Lecythidaceae & Eschweilera pedicellata (Rich.) S.A.Mori & 1 \\
\hline \multirow[t]{2}{*}{ Melastomataceae } & Bellucia grossularioides (L.) Triana & 1 \\
\hline & Miconia stenostachya DC. & 5 \\
\hline Menispermaceae & Abuta grandifolia (Mart.) Sandwith & 1 \\
\hline Moraceae & Pseudolmedia macrophylla Trécul & 1 \\
\hline Myristicaceae & Virola sp. & 1 \\
\hline Quiinaceae & Touroulia guianensis Aubl. & 2 \\
\hline Rubiaceae & Palicourea cf. corymbifera (Müll. Arg.) Standl. & 3 \\
\hline Salicaceae & Casearia sylvestris $\mathrm{Sw}$. & 1 \\
\hline \multirow[t]{3}{*}{ Sapindaceae } & Cupania scrobiculata Rich. & 1 \\
\hline & Talisia cerasina (Benth.) Radlk. & 1 \\
\hline & Talisia marleneana (Guarim) Acev.-Rodr. & 2 \\
\hline \multirow[t]{2}{*}{ Sapotaceae } & Pouteria virescens Baehni & 1 \\
\hline & Pouteria sp. & 3 \\
\hline Siparunaceae & Siparuna sp. & 1 \\
\hline Turneraceae & Turnera sp. & 1 \\
\hline $\mathrm{N}=22$ & $\mathrm{~N}=38$ (+ 1 subsp. $)$ & $\mathrm{N}=76$ \\
\hline
\end{tabular}

Table 2. Richness of insect galls in different Neotropical localities.

Tabela 2. Riqueza de galhas de insetos em diferentes localidades neotropicais.

\begin{tabular}{|c|c|c|c|c|c|}
\hline Locality & $\begin{array}{c}\text { Number }(\mathrm{Nr}) \text { of gall } \\
\text { morphotypes }\end{array}$ & $\begin{array}{l}\text { Nr. galled plant } \\
\text { families }\end{array}$ & $\begin{array}{c}\text { Nr. galled plant } \\
\text { genera }\end{array}$ & $\begin{array}{c}\text { Nr. galled plant } \\
\text { species }\end{array}$ & $\begin{array}{c}\text { Medium number of gall per } \\
\text { host plant species }\end{array}$ \\
\hline $\mathrm{PN}$ & 50 & 48 & 35 & 28 & 1.0 \\
\hline $\mathrm{CA}$ & 1038 & 491 & 185 & 48 & 2.1 \\
\hline $\mathrm{TA}$ & 54 & 27 & 22 & 10 & 2.0 \\
\hline PT & 133 & 75 & 60 & 37 & 1.7 \\
\hline $\mathrm{GO}$ & 34 & 20 & 17 & 12 & 1.7 \\
\hline SRPQ & 35 & 24 & 23 & 15 & 1.5 \\
\hline $\mathrm{BE}$ & 233 & 123 & 89 & 48 & 1.9 \\
\hline RBPS & 36 & 22 & 21 & 16 & 1.6 \\
\hline $\mathrm{MC}$ & 101 & 53 & 42 & 32 & 2.1 \\
\hline GR & 43 & 25 & 21 & 19 & 1.7 \\
\hline
\end{tabular}

AC-Arraial do Cabo (Rio de Janeiro, Brazil); BE-Bertioga (São Paulo, Brazil); CA-Central Amazonia (Amazonas, Brazil); CP-Serra do Cipó (Minas Gerais, Brazil); EP-Espinhaço (Brazil); FB-Fazenda Bulcão (Minas Gerais, Brazil); GO-Goiania (Goiás, Brazil); GR-Grumari (Rio de Janeiro, Brazil); JU-Jurubatiba (Rio de Janeiro, Brazil); MC-Maricá and Carapebus (Rio de Janeiro, Brazil); PEPCV-Parque Estadual Paulo César Vinha (Espírito Santo, Brazil); PN-Panama; PT-Pantanal (Mato Grosso do Sul, Brazil); RBPS-Reserva Biológica da Praia do Sul (Rio de Janeiro, Brazil); SRPQ-Santa Rita do Passa Quatro (São Paulo, Brazil); SSJ-Serra de São José (Minas Gerais, Brazil); TA (Tapajós, Pará, Brazil).

AC-Arraial do Cabo (Rio de Janeiro, Brasil); BE-Bertioga (São Paulo, Brasil); CA-Amazonia Central (Amazonas, Brasil); CP-Serra do Cipó (Minas Gerais, Brasil); EP-Espinhaço (Brasil); FB-Fazenda Bulcão (Minas Gerais, Brasil); GO-Goiânia (Goiás, Brasil); GR-Grumari (Rio de Janeiro, Brasil); JUJurubatiba (Rio de Janeiro, Brasil); MC-Maricá e Carapebus (Rio de Janeiro, Brasil); PEPCV-Parque Estadual Paulo César Vinha (Espírito Santo, Brasil); PN-Panamá; PT-Pantanal (Mato Grosso do Sul, Brasil); RBPS-Reserva Biológica da Praia do Sul (Rio de Janeiro, Brasil); SRPQ-Santa Rita do Passa Quatro (São Paulo, Brasil); SSJ-Serra de São José (Minas Gerais, Brasil); TA (Tapajós, Pará, Brasil). 
Table 2. Continued...

\begin{tabular}{lcccc}
\hline Locality & $\begin{array}{c}\text { Number (Nr) of gall } \\
\text { morphotypes }\end{array}$ & $\begin{array}{c}\text { Nr. galled plant } \\
\text { families }\end{array}$ & $\begin{array}{c}\text { Nr. galled plant } \\
\text { genera }\end{array}$ & $\begin{array}{c}\text { Nr. galled plant } \\
\text { species }\end{array}$ \\
\hline AC & 41 & 26 & 22 & 19 \\
JU & 99 & 25 & 36 & 40 \\
PEPCV & 38 & 21 & 19 & 17 \\
FB & 29 & 24 & 18 & 12 \\
SSJ & 137 & 73 & 47 & 30 \\
CP & 90 & 50 & 37 & 1.5 \\
EP & 384 & 142 & 75 & 1.9 \\
\hline
\end{tabular}

AC-Arraial do Cabo (Rio de Janeiro, Brazil); BE-Bertioga (São Paulo, Brazil); CA-Central Amazonia (Amazonas, Brazil); CP-Serra do Cipó (Minas Gerais, Brazil); EP-Espinhaço (Brazil); FB-Fazenda Bulcão (Minas Gerais, Brazil); GO-Goiania (Goiás, Brazil); GR-Grumari (Rio de Janeiro, Brazil); JU-Jurubatiba (Rio de Janeiro, Brazil); MC-Maricá and Carapebus (Rio de Janeiro, Brazil); PEPCV-Parque Estadual Paulo César Vinha (Espírito Santo, Brazil); PN-Panama; PT-Pantanal (Mato Grosso do Sul, Brazil); RBPS-Reserva Biológica da Praia do Sul (Rio de Janeiro, Brazil); SRPQ-Santa Rita do Passa Quatro (São Paulo, Brazil); SSJ-Serra de São José (Minas Gerais, Brazil); TA (Tapajós, Pará, Brazil).

AC-Arraial do Cabo (Rio de Janeiro, Brasil); BE-Bertioga (São Paulo, Brasil); CA-Amazonia Central (Amazonas, Brasil); CP-Serra do Cipó (Minas Gerais, Brasil); EP-Espinhaço (Brasil); FB-Fazenda Bulcão (Minas Gerais, Brasil); GO-Goiânia (Goiás, Brasil); GR-Grumari (Rio de Janeiro, Brasil); JUJurubatiba (Rio de Janeiro, Brasil); MC-Maricá e Carapebus (Rio de Janeiro, Brasil); PEPCV-Parque Estadual Paulo César Vinha (Espírito Santo, Brasil); PN-Panamá; PT-Pantanal (Mato Grosso do Sul, Brasil); RBPS-Reserva Biológica da Praia do Sul (Rio de Janeiro, Brasil); SRPQ-Santa Rita do Passa Quatro (São Paulo, Brasil); SSJ-Serra de São José (Minas Gerais, Brasil); TA (Tapajós, Pará, Brasil).

$(\mathrm{N}=8)$, and Tetragastris Gaertn $(\mathrm{N}=6)$ were the plant genera and Protium sagotianum Marchand ( $\mathrm{N}=7)$, Tetragastris panamensis $(\mathrm{N}=6)$, and Miconia stenostachya $\mathrm{DC} .(\mathrm{N}=5)$ were the plant species with the greatest diversity of galls (Table 1).

The majority of these galls occurred on leaves $(\mathrm{N}=61)$. Stems $(\mathrm{N}=15)$ and buds $(\mathrm{N}=1)$ were also attacked by galling species. Otherwise, galls on flower and fruit were not found.

Twenty five morphotypes were induced by Cecidomyiidae (Diptera) and one by Lepidoptera. The other inducers could not be determined as gall samples were collected already unoccupied $(\mathrm{N}=46)$, or occupied only by predators $(\mathrm{N}=1)$ or parasitoids $(\mathrm{N}=3)$.

Besides the galling species, other dwellers belong to Coleoptera, Diptera, Hymenoptera, Psocoptera and Thysanoptera were found. They were obtained from one, two, eight, one and two gall morphotypes, respectively. They showed diversified habits, acting as inquilines (Coleoptera, Diptera: Cecidomyiidae, Contarinia sp. and Thysanoptera), predators (Diptera: Cecidomyiidae: Lestodiplosini), parasitoids (Hymenoptera) or successor (Psocoptera). Inquilines were obtained from 13 gall morphotypes (17\%), parasitoids from three (4\%) and predators and successor from a single one.

Data on insect galls are presented here under host plant family, genus and species in alphabetical order. They include morphological characterization (plant organ, shape, color, presence/absence of trichomas, number of internal chamber and galler), as well as information of other dwellers (predators, parasitoids, inquilines, and sucessors) and previous gall records on the same host plant species, genus or family in the Neotropical region.

\section{ACHARIACEAE}

Lindackeria atifolia Benth.

Yellow, glabrous, one-chambered, circular leaf blade gall. Galler: not determined. New record of plant host family.

\section{BIGNONIACEAE}

\section{Arrabidaea sp.}

Greenish or yellowish, micro pubescent, one-chambered, elliptical leaf galls on veins and petiole (Figure 1). Galler: Cecidomyiidae. Other dwellers: Coleoptera.

Yellowish, glabrous, one-chambered, globose leaf galls (Figure 2). Galler: not determined.
Tavares (1918), Maia (2001) and Santos et al ( 2010) described some galls on A. conjugata (Vell.) Mart. and Arrabidaea sp.

\section{Memora magnifica (Mart. ex DC.) Bureau}

Brown, glabrous, woody, multichambered, elliptical leaf gall on midvein (Figure 3). Galler: Neolasioptera sp. (Cecidomyiidae). Other dwellers: Hymenoptera (parasitoids). New record of host plant species.

Oda (2006) described a single gall morphotype on Memora flavida.

\section{BORAGINACEAE}

\section{Cordia scabrifolia A.DC.}

Whitish, glabrous, multichambered, elliptical stem gall (Figure 4). Galler: Cecidomyiidae. Other dwellers: Hymenoptera (parasitoids). New record of host plant species.

\section{Cordia sp.}

Yellowish, glabrous, one-chambered, globose leaf gall (Figure 5). Galler: not determined.

Tavares (1925), Möhn (1959, 1964, 1975), Fernandes et al. (1988), Wünsche (1979), Maia (2001), Fernandes \& Negreiros (2006), Oda (2006), Julião (2007), Maia et al. (2008), and Coelho et al. (2009) described insect galls on Cordia alba, C. alliodora, C. cana, C. currassavica (Jacq.) Roem. \& Schult. ( = C. verbenacea), C. dentata, C. sagotti Johnst, C. sellowiana Cham., C. subtruncata, C. trichotoma (Vell.) Arráb. ex Steud., and Cordia sp.

\section{BURSERACEAE}

Protium giganteum Engl.

Green, glabrous, one-chambered, conical leaf gall (Figure 6). Galler: Cecidomyiidae. New host plant record.

Protium cf. giganteum Engl. var. giganteum

Green, glabrous, one-chambered, marginal leaf roll (Figure 7). Galller: not determined. 


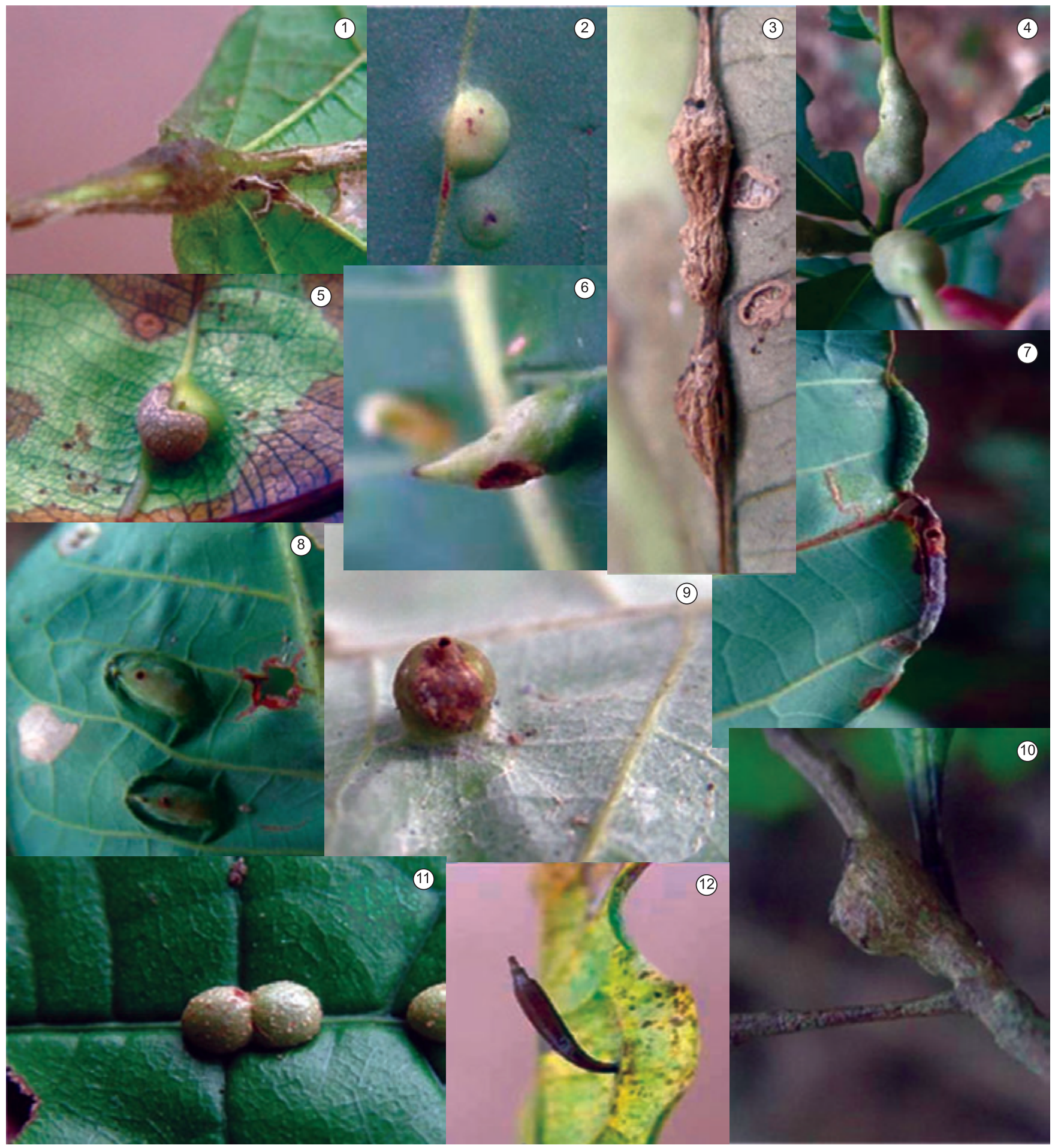

Figures 1-12. Insect galls found in Porto de Trombetas (Pará, Brazil). On Arrabidaea sp.: 1) elliptical leaf gall; 2) globose leaf gall; on Memora magnifica: 3) elliptical midvein swelling; on Cordia scabrifolia: 4) elliptical stem gall; on Cordia sp.: 5) globose leaf gall; on Protium giganteum: 6) conical leaf gall; on Protium cf. giganteum var. giganteum: 7) marginal leaf roll; 8) elliptical leaf gall: 9) globose leaf gall with apical projection; on Protium paniculatum: 10) stem swelling; on Protium sagotianum: 11) globose leaf gall; 12) elliptical, pedunculate leaf gall.

Figuras 1-12. Galhas de insetos encontradas em Porto de Trombetas (Pará, Brasil). Em Arrabidaea sp.: 1) galha foliar elíptica; 2) galha foliar globosa; em Memora magnifica: 3) intumescência elíptica da nervura central; em Cordia scabrifolia: 4) galha caulinar elíptical; em Cordia sp.: 5) galha foliar globosa; em Protium giganteum: 6) galha foliar cônica; em Protium cf. giganteum var. giganteum: 7) enrolamento da margem foliar; 8) galha foliar elíptica; 9) galha foliar globosa com projeção apical; em Protium paniculatum: 10) intumescência caulinar; em Protium sagotianum: 11) galha foliar globosa; 12) galha foliar elíptica pedunculada. 
Green, glabrous, one-chambered, elliptical leaf gall (Figure 8). Galler: Cecidomyiidae.

Green, glabrous, one-chambered, ovoid leaf gall. Galler: not determined.

Green, glabrous, one-chambered, globose leaf gall with apical projection (Figure 9). Galler: not determined.

\section{Protium paniculatum Engl.}

Brown, glabrous, one-chambered, unilateral, globose stem swelling (Figure 10). Galler: not determined.

Julião (2007) recorded one gall morphotype on the same host plant species.

\section{Protium sagotianum Marchand}

Brownish, glabrous, one-chambered, globose leaf gall (Figure 11). Galler: not determined.

Brownish, glabrous, one-chambered, pedunculate, elliptical leaf gall (Figure 12). Galler: not determined.

Green, glabrous, one-chambered, globose leaf gall (Figure 13). Galler: not determined.

Reddish, glabrous, one-chambered, globose leaf gall (Figure 14). Galler: not determined. Other dwellers: Lestodiplosini (Cecidomyiidae) (predator).

Green, glabrous, one-chambered, marginal leaf roll (Figure 15). Galler: Cecidomyiidae. Other dwellers: Thysanoptera.

Green, glabrous, one-chambered, conical leaf gall (Figure 16). Galler: Cecidomyiidae.

Houard (1933) listed an insect leaf gall on the same species of Protium, but morphologically different.

Protium spruceanum (Benth.) Engl.

Green, glabrous, one-chambered, marginal leaf roll (Figure 17). Galler: Cecidomyiidae. Other dweller: Contarinia sp. (Cecidomyiidae)

Green, glabrous, one-chambered, conical leaf gall. Galler: not determined.

Yellow, one-chambered, conical leaf gall (Figure 18). Galler: Cecidomyiidae

Brown, one-chambered, globose stem swelling. Galler: not determined.

Tavares (1922), Maia (2001), Oda (2006), Julião (2007), and NievesAldrey et al. (2008) described several gall morphotypes on 10 different species of Protium.

\section{Tetragastris panamensis (Engl.) Kuntze}

Yellow, glabrous, one-chambered, circular leaf gall (Figure 19). Galler: not determined.

Green, glabrous, one-chambered, globose vein swelling (Figure 20).

Galler: not determined.
Yellow, glabrous, one-chambered, parenchymatical leaf gall (Figure 21). Galler: Cecidomyiidae.

Brown, one-chambered, globose stem and petiole swelling (Figure 22). Galler: not determined.

Brown, one-chambered, bulbous stem swelling (Figure 23). Galler: not determined.

Brown, multi-chambered, elliptical stem swelling (Figure 24). Galler: not determined.

Nieves-Aldrey et al. (2008) described a leaf gall induced by Psyllidae (Hemiptera) on this same plant.

\section{CECROPIACEAE}

Pourouma guianensis Aubl.

Brown, glabrous, one-chambered, ovoid leaf gall. Galler: not determined.

Brown, glabrous, one-chambered, conical leaf gall (Figure 25). Galler: Cecidomyiidae.

Green, one-chambered, midvein swelling (Figure 26). Galler: not determined.

Julião (2007) recorded two gall morphotypes on this same host plant species. Rübsaamen (1908) and Julião (2007) recorded galls on five other species of Pourouma.

\section{CHRYSOBALANACEAE}

\section{Licania blackii Prance}

Brown, one-chambered, parenchymatical leaf gall (Figure 27). Galler: not determined.

\section{Licania longistyla (Hook. f.) Fritsch}

Red, hairy, one-chambered, globose leaf gall (Figure 28). Galler: Cecidomyiidae.

Gagné \& Hibbard (1996), Maia \& Fernandes (2004), Oda (2006), Julião (2007), Maia et al. (2008), Nieves-Aldrey et al. (2008), and Santos et al. (2010) recorded several gall morphotypes on 26 other species of Licania.

\section{EUPHORBIACEAE}

Alaeophora sp.

Green, glabrous, one-chambered, fusiform, stem swelling. Galler: Cecidomyiidae. New record of host plant species.

\section{FABACEAE}

Inga cayennensis Sagot ex Benth.

Brown, glabrous, one-chambered, parenchymatical leaf gall (Figure 29). Galler: not determined.

Inga coriacea var. leptopus (Benth.) J. F. Macbr.

Green, glabrous, one-chambered, elliptical midvein swelling (Figure 30). Galler: not determined. 


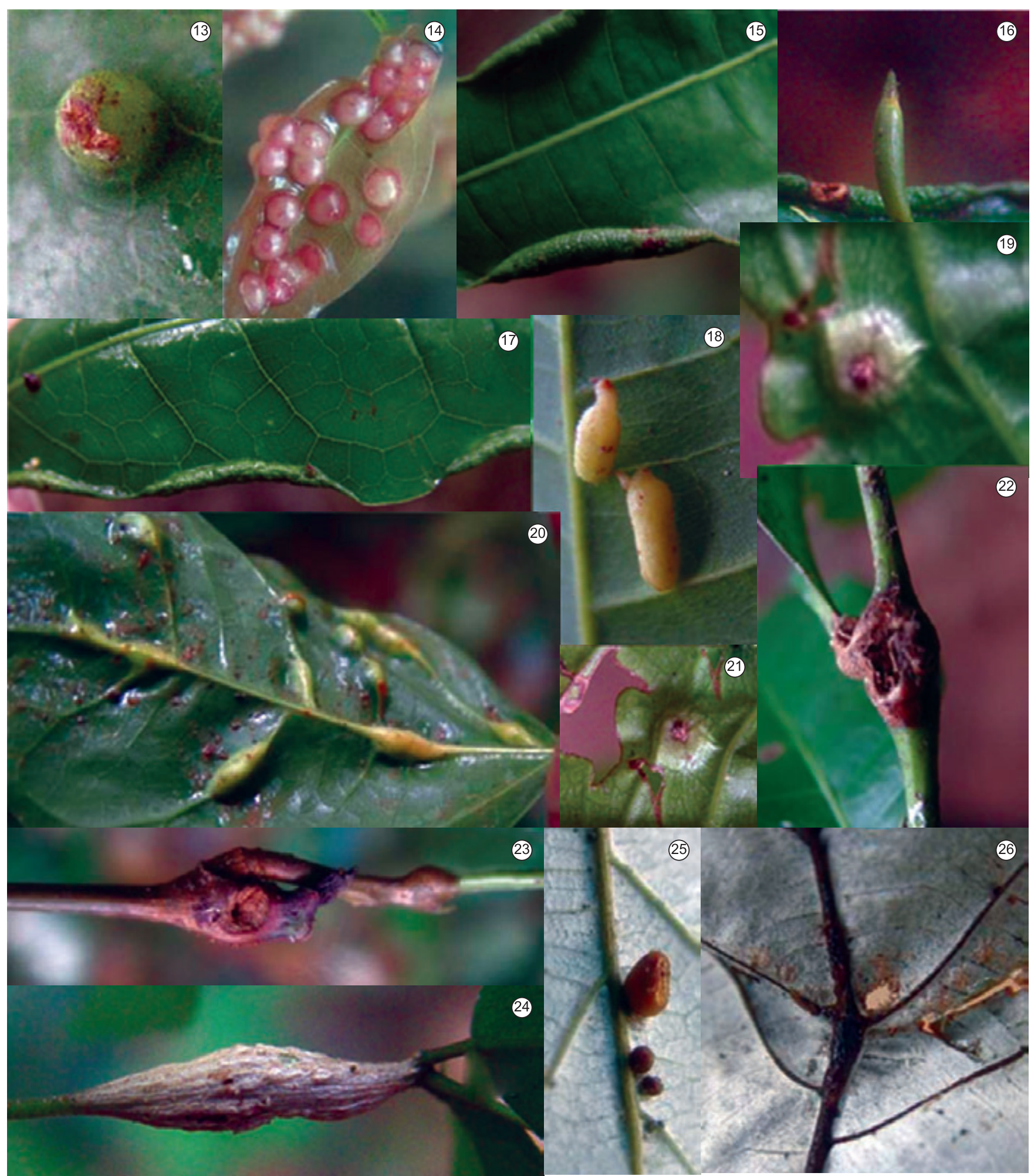

Figures 13-26. Insect galls found in Porto de Trombetas (Pará, Brazil). On Protium sagotianum.: 13) globose leaf gall (green); 14) globose leaf gall (reddish); 15) marginal leaf roll; 16) conical leaf gall; on Protium spruceanum: 17) marginal leaf roll; 18) conical leaf gall; on Tetragastris panamensis: 19) circular leaf gall; 20) vein swelling; 21) parenchymatical leaf gall; 22) stem and petiole swelling; 23) bulbous stem swelling; 24) elliptical stem swelling; on Pourouma guianensis: 25) conical leaf gall; 26) midvein swelling.

Figuras 13-26. Galhas de insetos encontradas em Porto de Trombetas (Pará, Brasil). Em Protium sagotianum.: 13) galha foliar globosa (verde); 14) galha foliar globosa (vermelha); 15) enrolamento da margem foliar; 16) galha foliar cônica; em Protium spruceanum: 17) enrolamento da margem foliar; 18) galha foliar cônica; em Tetragastris panamensis: 19) galha foliar circular; 20) intumescência da nervura; 21) galha foliar parenquimática; 22) intumescência do caule e pecíolo; 23) intumescência bulbosa do caule; 24) intumescência elíptica do caule; em Pourouma guianensis: 25) galha foliar cônica; 26) intumescência da nervura central. 


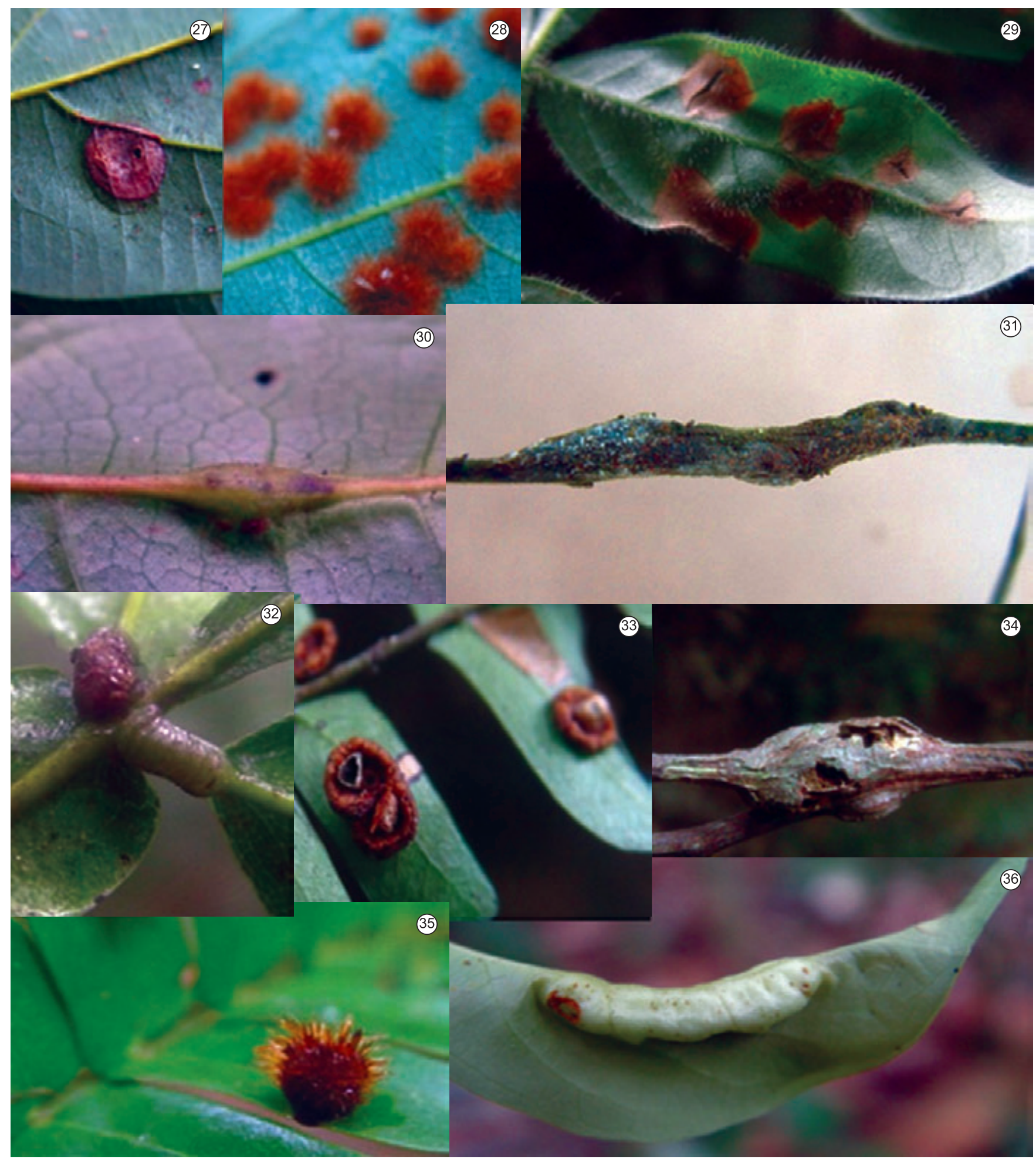

Figures 27-36. Insect galls found in Porto de Trombetas (Pará, Brazil). On Licania blackii.: 27) parenchimatical leaf gall; on Licani longistyla: 28) globose leaf gall; on Inga cayennensis: 29) parenchymatical leaf gall; on Inga coriacea var. leptopus: 30) elliptical midvein swelling; on Inga rubiginosa: 31) bulbous stem swelling; on Inga umbellifera: 32) midvein swelling; on Zygia racemosa: 33) discoid leaf gall; 34) bulbous stem swelling; 35) globose leaf gall; on Lacistema polystachyum: 36 ) coalescent leaf gall.

Figuras 27-36. Galhas de insetos encontradas em Porto de Trombetas (Pará, Brasil). Em Licania blackii.: 27) galha foliar parenquimática; em Licania longistyla: 28) galha foliar globosa; em Inga cayennensis: 29) galha foliar parenquimática; em Inga coriacea var. leptopus: 30) intumescência elíptica da nervura central; em Inga rubiginosa: 31) intumescência bulbosa do caule; em Inga umbellifera: 32) intumescência da nervura central; em Zygia racemosa: 33) galha foliar discóide; 34) intumescência bulbosa do caule; 35) galha foliar globosa; em Lacistema polystachyum: 36) galha foliar coalescente. 
Inga rubiginosa (Rich.) DC.

Brown, hairy, one-chambered, globose leaf gall. Galler: Cecidomyiidae.

Brown, multi-chambered, bulbous stem swelling (Figure 31). Galler: Cecidomyiidae.

Green, one-chambered, elliptical vein swelling. Galler: not determined.

Julião (2007) recorded two gall morphotypes on this same host plant species.

Inga umbellifera (Vahl) Steud.

Brown, hairy, one-chambered, globose leaf gall. Galler: not determined.

Yellow, glabrous, one-chambered, globoid leaf gall. Galler: Cecidomyiidae.

Brown, glabrous, one-chambered, midvein swelling (Figure 32). Galler: Cecidomyiidae.

Julião (2007) recorded six gall morphotypes on this same host plant species. Rübsaamen (1907), Tavares (1920), Möhn (1964, 1975), Fernandes et al. (1988), Maia ( 2001), Maia et al. (2002), Fernandes \& Negreiros (2006), Julião (2007), Maia et al. (2008), NievesAldrey et al. (2008), and Santos et al. (2010) described several galls on 24 other species of Inga spp.

\section{Zygia racemosa (Ducke) Barneby \& J.W.Grimes}

Brown, glabrous, one-chambered, discoid leaf gall (Figure 33). Galler not determined.

Brown, multi-chambered, bulbous stem swelling (Figure 34). Galler not determined.

Red, hairy, one-chambered, globose leaf gall (Figure 35). Galler: Cecidomyiidae.

Julião (2007) recorded five gall morphotypes on this same plant species.

\section{LACISTEMATACEAE}

Lacistema polystachyum Schnizl.

Green, glabrous, coalescent, multi-chambered leaf gall (Figure 36). Galler: Cecidomyiidae.

Julião (2007) and Nieves-Aldrey et al. (2008) described some gall morphotypes on Lacistema aggregatum (Bergius) Rusby.

\section{LAURACEAE}

Aniba burchellii Kosterm.

Brown, glabrous, one-chambered, globose leaf gall. Galler: not determined.

Aniba sp.

Brown, hairy, one-chambered, globose leaf gall (Figure 37). Galler: Macroporpa sp. (Cecidomyiidae).
Julião (2007) recorded some gall morphotypes on three species of this genus: Aniba burchelii Kosterm., A. canelilla (H.B.H.) Mez., and $A$. ferrea Kubitzki.

\section{LECYTHIDACEAE}

Eschweilera pedicellata (Rich.) S.A. Mori

Yellow, glabrous, one-chambered, globose leaf gall (Figure 38). Galler: Contarinia sp. (Cecidomyiidae). Dwellers: Thysanoptera and Hymenoptera.

Julião (2007) recorded several gall morphotypes on other 15 species of Eschweilera.

\section{MELASTOMATACEAE}

Bellucia grossularioides (L.) Triana

Green, grabrous, one-chambered, globoid stem, petiole and midvein swelling (Figure 39). Galler: not determined. New record of host plant genus.

Miconia stenostachya DC.

Brown, glabrous, one-chambered, globoid leaf gall (Figure 40). Galler: not determined. Dweller: Hymenoptera.

Green, glabrous, one-chambered, elliptical veins swelling (Figure 41). Galler: not determined.

Brown, glabrous, one-chambered, globoid stem swelling (Figure 42). Galler: not determined.

Yellowish, hairy, one-chambered, globose leaf gall (Figure 43). Galler: not determined. Dwellers: Hymenoptera and Psocoptera.

Green, glabrous, one-chambered, parenchymatical leaf gall (Figure 44). Galler: Cecidomyiidae.

Brown, glabrous, one-chambered, bulbous stem swelling (Figure 45). Galler: Lepidoptera.

Rübsaamen (1907), Tavares (1917, 1925), Houard (1933), Maia (2001), Maia \& Fernandes (2004), Oda (2006), Julião (2007), and Maia et al. (2008) recorded galls on 15 other species of Miconia.

\section{MENISPERMACEAE}

Abuta grandifolia (Mart.) Sandwith

Brown, glabrous, one-chambered, unilateral stem swelling (Figure 46). Galler: not determined. New record of host plant genus.

Möhn (1964) described a Cecidomyiidae gall morphotype on Cissampelos pareira. This was the single previously known record of insect gall on Menispermaceae.

\section{MORACEAE}

\section{Pseudolmedia macrophylla Trécul}

Yellow, glabrous, one-chambered, globose leaf gall. Galler: not determined.

Houard (1924) described a hairy, globose gall induced by insect on P. sagoti. 


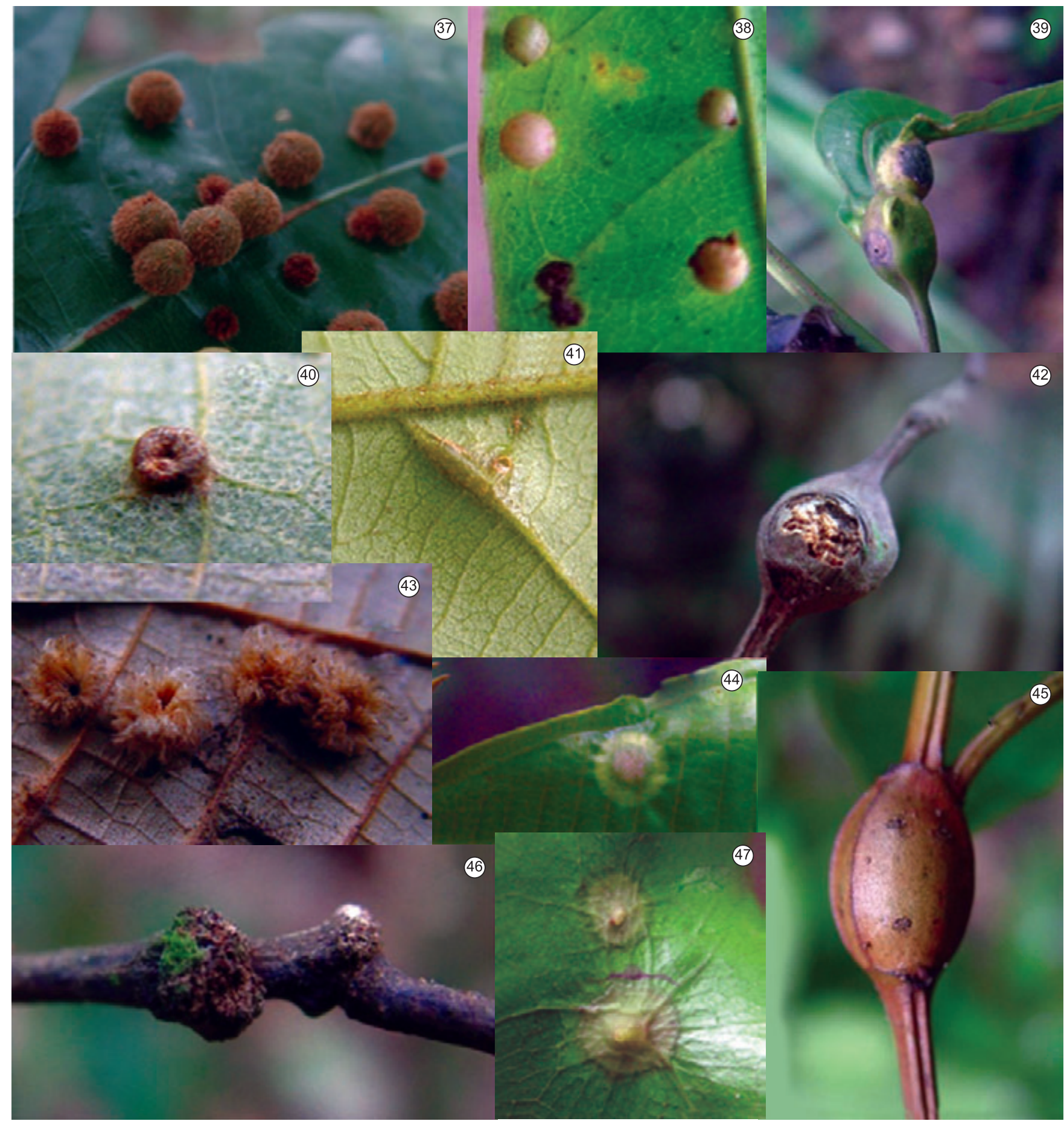

Figures 37-47. Insect galls found in Porto de Trombetas (Pará, Brazil). On Aniba sp.: 37) globose leaf gall; on Eschweilera pedicellata: 38) globose leaf gall; on Bellucia grossularioides: 39) globoid stem, petiole and midvein swelling; on Miconia stenostachya: 40) globoid leaf gall; 41) veins swelling; 42) globoid stem swelling; 43) globose leaf gall; 44) parenchymatical leaf gall; 45) bulbous stem swelling; on Abuta grandifolia: 46) stem swelling; on Virola sp.: 47) parenchymatical leaf gall.

Figuras 37-47. Galhas de insetos encontradas em Porto de Trombetas (Pará, Brasil). Em Aniba sp.: 37) galha foliar globosa; em Eschweilera pedicellata: 38) galha foliar globosa; em Bellucia grossularioides: 39) intumescência globóide da nervura central, pecíolo e caule; em Miconia stenostachya: 40) galha foliar globóide; 41) intumescência das nervuras; 42) intumescência globóide do caule; 43) galha foliar globosa; 44) galha foliar parenquimática; 45) intumescência bulbosa do caule; em Abuta grandifolia: 46) intumescênciado caule; em Virola sp.: 47) galha foliar parenquimática. 


\section{MYRISTICACEAE}

Virola sp.

Yellow, glabrous, one-chambered, parenchymatical leaf gall (Figure 47). Galler: not determined.

Nieves-Aldrey et al. (2008) described a Cecidomyiidae leaf gall on the same plant genus.

\section{QUIINACEAE}

Touroulia guianensis Aubl.

Brown, one-chambered, midvein swelling (Figure 48). Galler: not determined.

Brown, glabrous, one-chambered, globoid leaf gall (Figure 49). Galler: not determined. New record of host plant family.

\section{RUBIACEAE}

Palicourea cf. corymbifera (Müll. Arg.) Standl.

Green, glabrous, one-chambered, conical leaf gall (Figure 50). Galler: Cecidomyiidae. Other dwellers: Hymenoptera (parasitoids).

Brown, one-chambered, lateral vein swelling. Galler: not determined.

Brown, one-chambered, bulbous stem swelling. Galler: not determined.

Gagné (1994) described two Cecidomyiidae gall morphotypes on Palicourea sp.

\section{SALICACEAE}

Casearia sylvestris Sw.

Brown, glabrous, one-chambered, fusiform stem swelling (Figure 51). Galler: not determined.

Rübsaamen (1905) described a Cecidomyiidae bud gall on Casearia sp., and Oda (2006) recorded a leaf gall whose inducer was not determined on Casearia sp. Nieves-Aldrey et al. (2008) described two kinds of Cecidomyiidae galls, one on leaf and the other on stem on C. commersoniana Cambess.

\section{SAPINDACEAE}

\section{Cupania scrobiculata Rich.}

Brown, glabrous, one-chambered, conical leaf gall. Galler: not determined.

Coelho et al. (2009) described a gall on C. vernalis Cambess., but the inducer was not identified.

\section{Talisia cerasina (Benth.) Radlk.}

Brown, glabrous, one-chambered, bulbous petiole swelling. Galler: not determined. New record of host plant genus.

\section{Talisia marleneana (Guarim) Acev.-Rodr.}

Green, glabrous, one-chambered, ovoid bud gall (Figure 52). Galler: not determined.
Green, glabrous, one-chambered, bulbous petiole swelling. Galler: not determined. New record of host plant species.

\section{SAPOTACEAE}

Pouteria virescens Baehni

Green, glabrous, one-chambered, parenchymatical leaf gall. Galler: not determined.

Pouteria sp.

Yellow, glabrous, one-chambered, conical leaf gall (Figure 53). Galler: Cecidomyiidae. Other dwellers: Hymenoptera (parasitoids).

Brown, glabrous, one-chambered, globose leaf gall (Figure 54). Galler: not determined.

Brown, glabrous, one-chambered, discoid leaf gall (Figure 55). Galler: not determined.

Rübsaamen (1908), Monteiro et al. (1993), Maia (2001), Fernandes \& Negreiros (2006), Julião (2007), and Nieves-Aldrey et al. (2008) recorded several gall morphotypes on 38 other species of Pouteria.

\section{SIPARUNACEAE}

Siparuna sp.

Brown, glabrous, rugose, one-chambered, discoid leaf gall (Figure 56). Galler: Cecidomyiidae.

Rübsaamen (1908) described two Eriophyidae galls on this genus of plant not determined species and Santos et al. (2010) recorded an insect gall morphotype on Siparuna guianensis Aubl.

\section{TURNERACEAE}

\section{Turnera sp.}

Green, glabrous, one-chambered, parenchymatical leaf gall. Galler: not determined. Dwellers: Hymenoptera.

Felt (1917) described a Cecidomyiidae stem gall on Piriqueta sp. This is the single previous gall record on Turneraceae.

\section{Discussion}

For the majority of insect gall species in Neotropical region, there are few data on geographic distributions, biology, ecology, phylogenetic relationships and life-history. This survey provides basic data from a poorly known region in Amazonian Forest.

Previous studies on insect galls in Brazilian biomes employed diverse sampling methods and involved different collecting effort which makes it difficult to compare species richness among sites. They have reported richness values ranging from 34 to 1038 (see references in Table 2). The number of gall morphotypes in Platô Bacaba is included in this range. Other two Amazonian Forest areas (Amazonia Central and Tapajós) showed 1028 and 54 gall morphotypes, respectively (Julião 2007, Oda 2006), but the collecting effort were not similar. These figures could indicate that Platô Bacaba does not comprise a great richness of insect galls.

The medium number of gall morphotypes found in Platô Bacaba does not differ from the values of other areas of Amazonian Forest, as well as of other ecosystems, such as "restinga" and "cerrado". These results can be explained by the fact of many host plant species support only one or two gall morphotypes while few 
Maia, V.C.

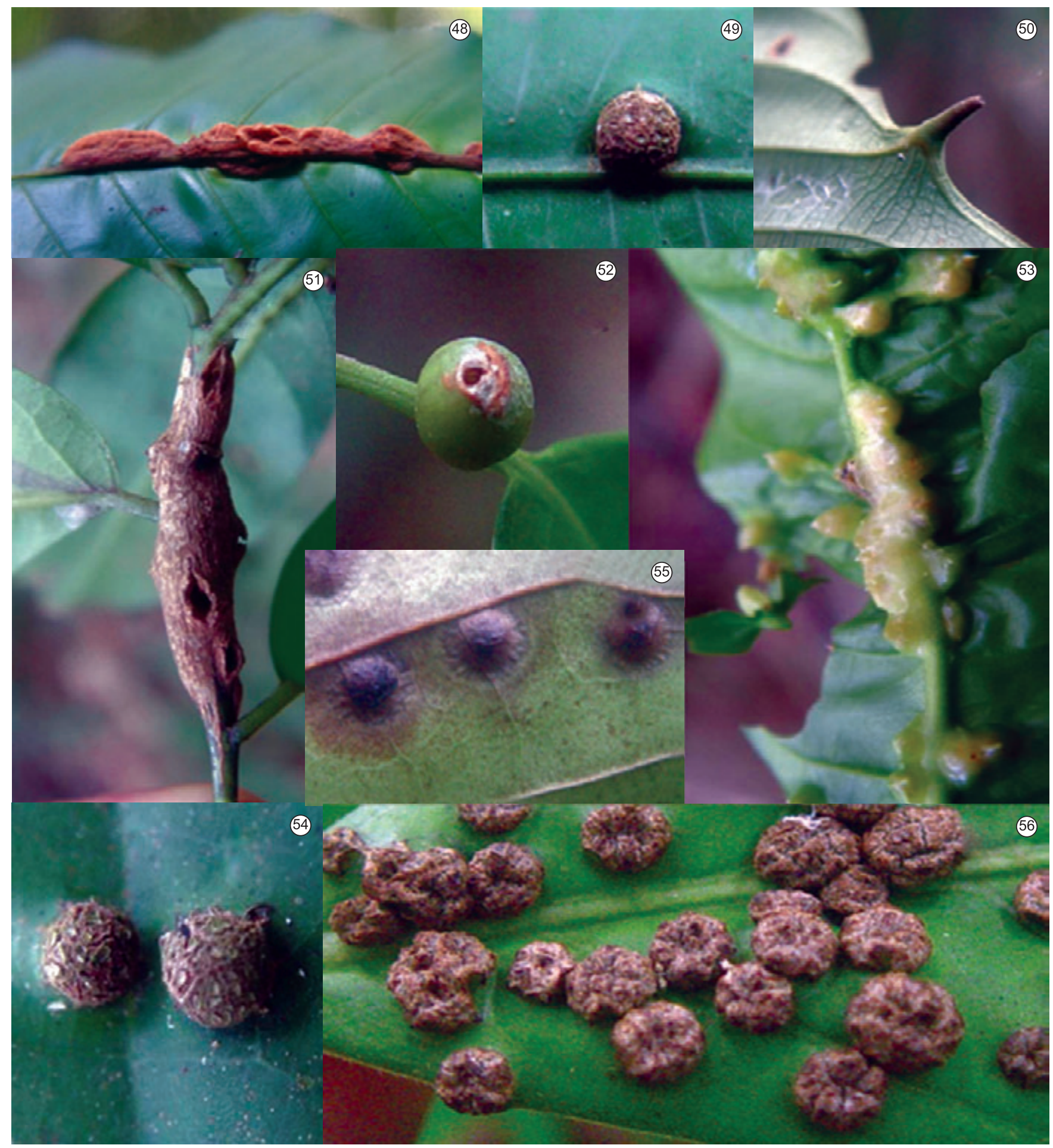

Figures 48-56. Insect galls found in Porto de Trombetas (Pará, Brazil). On Touroulia guianensis.: 48) midvein swelling; 49) globoid leaf gall; on Palicourea cf. corymbifera: 50) conical leaf gall; on Casearia sylvestris: 51) fusiform stem swelling; on Talisia marleneana: 52) ovoid bud gall; on Pouteria sp.: 53) conical leaf gall; 54) globose leaf gall; 55) discoid leaf gall; on Siparuna sp.: 56) discoid leaf gall.

Figures 48-56. Galhas de insetos encontradas em Porto de Trombetas (Pará, Brasil). Em Touroulia guianensis.: 48) intumescência da nervura central; 49) galha foliar globóide; em Palicourea cf. corymbifera: 50) galha foliar cônica; em Casearia sylvestris: 51) intumescência fusiforme do caule; em Talisia marleneana: 52) ovóide da gema; em Pouteria sp.: 53) galha foliar cônica; 54) galha foliar globosa: 55) galha foliar discóide; em Siparuna sp.: 56) galha foliar discóide. 
others, known as super host, support a highest number. Some plant characters qualify or influence the species as superhost: plants with wide morphogenical potential comprise a greatest number of gall morphotypes (Oliveira et al. 2008); the presence of tannins influences positively the richness of galls (Espírito-Santo et al. 1999); the plant architecture hypothesis states that the physical structure of the aerial parts of the host plant influences the community structure of herbivorous insects. A pattern of increased insect herbivore species richness and/or abundance with architecture host plant has been supported by several studies (Lara et al. 2008). Other factors, such as age, abundance and distribution of the plant species can also influence the gall richness, but they were not studied in this survey.

Leaves were the most galled plant organ in Platô Bacaba. The high diversity of leaf galls is observed in all Neotropical biomas (Table 3), as well as in the world (Mani 1964), probably because leaves represent an abundant and frequently renewable resource, with undifferentiated meristematic cells which are essential to gall growth. The predominance of glabrous and one-chambered galls in

Table 3. Distribution of insect galls per attacked plant organ in different Neotropical localities.

Tabela 3. Distribuição de galhas de insetos por órgão vegetal atacado em diferentes localidades neotropicais.

\begin{tabular}{lccccccc}
\hline Locality & $\begin{array}{c}\text { Leaf } \\
(\boldsymbol{\%})\end{array}$ & $\begin{array}{c}\text { Stem } \\
(\boldsymbol{\%})\end{array}$ & $\begin{array}{c}\text { Bud } \\
(\boldsymbol{\%})\end{array}$ & $\begin{array}{c}\text { Fruit } \\
(\boldsymbol{\%})\end{array}$ & $\begin{array}{c}\text { Flower } \\
(\boldsymbol{\%})\end{array}$ & $\begin{array}{c}\text { Tendril } \\
(\boldsymbol{\%})\end{array}$ & $\begin{array}{c}\text { Adventicuous } \\
\text { root }(\boldsymbol{\%})\end{array}$ \\
\hline PN & 96.0 & 2.0 & 2.0 & - & - & - & - \\
CA & 85.0 & 10.7 & 3.0 & $<1.0$ & $<1.0$ & - & - \\
TA & 94.0 & 6.0 & - & - & - & - & - \\
PT & 69.2 & 17.3 & 12.8 & - & $<1.0$ & - & - \\
GO & 79.0 & 21.0 & - & - & - & - & - \\
SRPQ & 77.0 & 28.5 & 2.8 & - & 2.8 & 2.8 & - \\
BE & 56.0 & 26.5 & 14.4 & - & 1.5 & $<1.0$ & $<1.0$ \\
RBPS & 64.0 & 25.0 & 14.0 & - & - & - & - \\
MC & 62.4 & 8.9 & 12.9 & 3.9 & 8.9 & 1.0 & - \\
GR & 62.8 & 16.3 & 11.6 & 4.6 & 9.3 & 2.3 & - \\
AC & 68.0 & 24.0 & 2.4 & - & 4.8 & - & - \\
PEPCV & 84.0 & 13.0 & - & 3.0 & - & - & - \\
FB & 72.0 & 28.0 & - & - & - & - & - \\
SSJ & 76.0 & 32.0 & 13.0 & - & 1.4 & - & - \\
CP & 63.0 & 34.0 & 1.0 & - & 1.0 & - & - \\
EP & 20.0 & 73.0 & - & - & $<1.0$ & - & - \\
\hline AC-AITa & &
\end{tabular}

AC-Arraial do Cabo (Rio de Janeiro, Brazil); BE-Bertioga (São Paulo, Brazil); CA-Central Amazonia (Amazonas, Brazil); CP-Serra do Cipó (Minas Gerais, Brazil); EP-Espinhaço (Brazil); FB-Fazenda Bulcão (Minas Gerais, Brazil); GO-Goiania (Goiás, Brazil); GR-Grumari (Rio de Janeiro, Brazil); MC-Maricá and Carapebus (Rio de Janeiro, Brazil); PEPCV-Parque Estadual Paulo César Vinha (Espírito Santo, Brazil); PN-Panama; PT-Pantanal (Mato Grosso do Sul, Brazil); RBPS-Reserva Biológica da Praia do Sul (Rio de Janeiro, Brazil); SRPQ-Santa Rita do Passa Quatro (São Paulo, Brazil); SSJ-Serra de São José (Minas Gerais, Brazil); TA (Tapajós, Pará, Brazil). Total exceeds 100\%, because some galls morphotypes were recorded on two plant organs or more.

AC-Arraial do Cabo (Rio de Janeiro, Brasil); BE-Bertioga (São Paulo, Brasil); CA-Amazonia Central (Amazonas, Brasil); CP-Serra do Cipó (Minas Gerais, Brasil); EP-Espinhaço (Brasil); FB-Fazenda Bulcão (Minas Gerais, Brasil); GO-Goiânia (Goiás, Brasil); GR-Grumari (Rio de Janeiro, Brasil); MC-Maricá e Carapebus (Rio de Janeiro, Brasil); PEPCV-Parque Estadual Paulo César Vinha (Espírito Santo, Brasil); PN-Panamá; PTPantanal (Mato Grosso do Sul, Brasil); RBPS-Reserva Biológica da Praia do Sul (Rio de Janeiro, Brasil); SRPQ-Santa Rita do Passa Quatro (São Paulo, Brasil); SSJ-Serra de São José (Minas Gerais, Brasil); TA (Tapajós, Pará, Brasil). O total excede 100\%, porque alguns morfotipos de galhas foram registrados em dois órgãos vegetais ou mais.
Platô Bacaba is also observed in all other studied localities (Table 4), indicating a general pattern.

The richest plant families in number of gall morphotypes in Platô Bacaba, as well as in restinga, cerrado, and other Amazonian Forest areas (Table 5) indicates that the greatest richness of galls is showed by the most speciose plant families of each area (Silva \& Pinheiro 2007, Carneiro et al. 2009, Julião 2007).

The plant genera with the highest diversity of galls in Platô Bacaba differ significantly in the number of described species. Protium, Inga and Tetragastris are known from 150, 400 and nine described species, respectively (Marques et al. 2010, Mata \& Felix 2007, Daly 1989), suggesting that the richness of insect galls does not depend upon the plant genus richness (a evidence against richness plant hypothesis, Lawton \& Schröder (1977)). Different results were found in restinga, cerrado and other Amazonian Forest areas, where the highest diversity of galls is showed by the most speciose plant genera (Table 6).

The great majority of the identified gallers belongs to Cecidomyiidae (Diptera). Cecidomyiidae are the most important galler not only in other Neotropical localities, but in all zoogeographic regions. Besides them, other insect orders comprise galling species, such as Lepidoptera, Thysanoptera, Hemiptera, Hymenoptera and Coleoptera, but in Platô Bacaba, these gallers were not found, except for Lepidoptera (Table 7).

Inquilines were more frequent than parasitoids and predators in Platô Bacaba. This result is very peculiar, as parasitoids are the most frequent and abundant natural enemies of galling species in restinga and cerrado areas, as well as throughout the world (Gagné 1994) (Tables 8 and 9). This pattern has not been previously documented in other Brazilian biomes or localities, and was, therefore, particularly interesting.

Table 4. Characterization of gall morphotypes (glabrous/pubescent, and one-/ multichambered) in different localities of the Neotropical region.

Tabela 4. Caracterização dos morfotipos de galha (glabro/pubescente unilocular/multilocular) em diferentes localidades da Região Neotropical.

\begin{tabular}{|c|c|c|c|c|}
\hline Locality & $\begin{array}{c}\text { Glabrous } \\
(\%)\end{array}$ & $\begin{array}{c}\text { Pubescent } \\
(\%)\end{array}$ & $\begin{array}{c}\text { One-chambered } \\
(\%)\end{array}$ & $\begin{array}{c}\text { Multi-chambered } \\
(\%)\end{array}$ \\
\hline $\mathrm{CA}$ & 95 & 5 & No data & No data \\
\hline PT & 89 & 11 & No data & No data \\
\hline GO & 76 & 24 & No data & No data \\
\hline SRPQ & 60 & 40 & 71 & 29 \\
\hline RBPS & 94 & 6 & 94 & 6 \\
\hline $\mathrm{MC}$ & 9 & 3 & 92 & 8 \\
\hline PEPCV & 89 & 11 & 52 & 48 \\
\hline FB & 79 & 21 & 86 & 14 \\
\hline $\mathrm{CP}$ & 77 & 23 & 77 & 23 \\
\hline $\mathrm{EP}$ & 94 & 06 & No data & No data \\
\hline
\end{tabular}

CA-Central Amazonia (Amazonas, Brazil); CP-Serra do Cipó (Minas Gerais, Brazil); EP-Espinhaço (Brazil); FB-Fazenda Bulcão (Minas Gerais, Brazil); GO-Goiania (Goiás, Brazil); MC-Maricá and Carapebus (Rio de Janeiro, Brazil); PEPCV-Parque Estadual Paulo César Vinha (Espírito Santo, Brazil); PT-Pantanal (Mato Grosso do Sul, Brazil); RBPS-Reserva Biológica da Praia do Sul (Rio de Janeiro, Brazil); SRPQ-Santa Rita do Passa Quatro (São Paulo, Brazil).

CA-Amazonia Central (Amazonas, Brasil); CP-Serra do Cipó (Minas Gerais, Brasil); EP-Espinhaço (Brasil); FB-Fazenda Bulcão (Minas Gerais, Brasil); GO-Goiânia (Goiás, Brasil); MC-Maricá e Carapebus (Rio de Janeiro, Brasil); PEPCV-Parque Estadual Paulo César Vinha (Espírito Santo, Brasil); PT-Pantanal (Mato Grosso do Sul, Brasil); RBPS-Reserva Biológica da Praia do Sul (Rio de Janeiro, Brasil); SRPQ-Santa Rita do Passa Quatro (São Paulo, Brasil). 
Maia, V.C.

Table 5. Plant families with the greatest insect galls richness in different Neotropical localities.

Tabela 5. Famílias de plantas hospedeiras com maior riqueza de galhas de insetos em diferentes localidades neotropicais.

\begin{tabular}{|c|c|c|c|c|c|c|c|c|c|c|c|c|c|c|c|c|}
\hline Plant family & PN & $\mathbf{C A}$ & TA & PT & GO & SRPQ & $\mathbf{B E}$ & RBPS & MC & GR & AC & PEPCV & FB & SSJ & $\mathbf{C P}$ & EP \\
\hline Asteraceae & - & - & - & - & - & - & $\mathrm{x}$ & $\mathrm{x}$ & - & - & - & - & - & $\mathrm{x}$ & $\mathrm{x}$ & $\mathrm{x}$ \\
\hline Bignoniaceae & $\mathrm{x}$ & - & - & $\mathrm{x}$ & - & - & - & - & - & - & - & - & - & - & - & - \\
\hline Burseraceae & - & - & $\mathrm{x}$ & - & - & - & - & - & $\mathrm{x}$ & - & - & - & - & - & - & - \\
\hline Erythroxylacaeae & - & - & - & - & - & - & - & - & - & $\mathrm{x}$ & - & - & - & - & - & - \\
\hline Euphorbiaceae & $\mathrm{x}$ & - & - & - & - & - & - & - & - & - & - & - & $\mathrm{x}$ & - & - & - \\
\hline Fabaceae & $\mathrm{x}$ & $\mathrm{x}$ & $\mathrm{x}$ & $\mathrm{x}$ & $\mathrm{x}$ & $\mathrm{x}$ & $\mathrm{x}$ & - & - & - & - & - & $\mathrm{x}$ & $\mathrm{x}$ & $\mathrm{x}$ & $\mathrm{x}$ \\
\hline Hippocrateaceae & - & - & - & $\mathrm{x}$ & - & - & - & - & - & - & - & - & - & - & - & - \\
\hline Lecythidaceae & - & $\mathrm{x}$ & $\mathrm{x}$ & - & - & - & - & - & - & - & - & - & - & - & - & - \\
\hline Malpighiaceae & - & - & - & - & - & $\mathrm{x}$ & - & - & - & - & - & - & - & - & - & $\mathrm{x}$ \\
\hline Melastomataceae & $\mathrm{x}$ & - & - & - & - & - & $\mathrm{x}$ & - & - & - & - & - & - & $\mathrm{x}$ & - & $\mathrm{x}$ \\
\hline Myrtaceae & $\mathrm{x}$ & - & - & - & - & $\mathrm{x}$ & $\mathrm{x}$ & $\mathrm{x}$ & $\mathrm{x}$ & $\mathrm{x}$ & $\mathrm{x}$ & $\mathrm{x}$ & - & $\mathrm{x}$ & $\mathrm{x}$ & - \\
\hline Nyctaginaceae & - & - & - & - & - & - & - & $\mathrm{x}$ & $\mathrm{x}$ & - & - & $\mathrm{x}$ & - & - & - & - \\
\hline Sapindaceae & - & - & - & $\mathrm{x}$ & - & - & - & - & - & - & - & - & - & - & - & - \\
\hline Sapotaceae & - & $\mathrm{x}$ & - & - & - & - & - & - & - & - & - & $\mathrm{x}$ & - & - & - & - \\
\hline Solanaceae & - & - & - & - & - & - & - & - & - & - & - & - & - & - & - & - \\
\hline Styracaceae & - & - & - & - & $\mathrm{x}$ & - & - & - & - & - & - & - & - & - & - & - \\
\hline Ulmaceae & - & - & - & - & $\mathrm{x}$ & - & - & - & - & - & - & - & - & - & - & - \\
\hline
\end{tabular}

AC-Arraial do Cabo (Rio de Janeiro, Brazil); BE-Bertioga (São Paulo, Brazil); CA-Central Amazonia (Amazonas, Brazil); CP-Serra do Cipó (Minas Gerais, Brazil); EPEspinhaço (Brazil); FB-Fazenda Bulcão (Minas Gerais, Brazil); GO-Goiania (Goiás, Brazil); GR-Grumari (Rio de Janeiro, Brazil); MC-Maricá and Carapebus (Rio de Janeiro, Brazil); PEPCV-Parque Estadual Paulo César Vinha (Espírito Santo, Brazil); PN-Panama; PT-Pantanal (Mato Grosso do Sul, Brazil); RBPS-Reserva Biológica da Praia do Sul (Rio de Janeiro, Brazil); SRPQ-Santa Rita do Passa Quatro (São Paulo, Brazil); SSJ-Serra de São José (Minas Gerais, Brazil); TA (Tapajós, Pará, Brazil). AC-Arraial do Cabo (Rio de Janeiro, Brasil); BE-Bertioga (São Paulo, Brasil); CA-Amazonia Central (Amazonas, Brasil); CP-Serra do Cipó (Minas Gerais, Brasil); EP-Espinhaço (Brasil); FB-Fazenda Bulcão (Minas Gerais, Brasil); GO-Goiânia (Goiás, Brasil); GR-Grumari (Rio de Janeiro, Brasil); MC-Maricá e Carapebus (Rio de Janeiro, Brasil); PEPCV-Parque Estadual Paulo César Vinha (Espírito Santo, Brasil); PN-Panamá; PT-Pantanal (Mato Grosso do Sul, Brasil); RBPS-Reserva Biológica da Praia do Sul (Rio de Janeiro, Brasil); SRPQ-Santa Rita do Passa Quatro (São Paulo, Brasil); SSJ-Serra de São José (Minas Gerais, Brasil); TA (Tapajós, Pará, Brasil).

Table 6. Distribution of the number of species by super host plant genera in different Brazilian localities and ecosystems.

Table 6. Distribuição do número de espécies por gêneros de planta considerados superhospedeiros de galhas em diferentes localidades do Brasil.

\begin{tabular}{lllll}
\hline \multicolumn{1}{c}{ Locality } & Ecosystem & Genera & \multicolumn{1}{c}{ Nr. species } & Reference \\
\hline Rio de Janeiro State & Restinga & Eugenia & 330 (Romagnolo \& Souza 2006) & Maia (2001) \\
São Paulo State & Restinga & Mikania & 450 (Ritter \& Waechter 2004) & Maia et al. (2008) \\
Minas Gerais & Cerrado & Baccharis & 320 (Heiden et al. 2007) & Fernandes et al. (1996) \\
& & Bauhinia & 300 (Vaz \& Tozzi 2005) & Carneiro et al. (2009) \\
Central Amazonia & Amazonian Forest & Pouteria & 330 (Alves-Araújo 2010) & Julião (2007) \\
& & Protium & 150 (Marques et al. 2010) & - \\
Platô Bacaba & Amazonian Forest & Protium & 150 (Marques et al. 2010) & Present manuscript \\
& & Inga & 400 (Mata \& Felix 2007) & - \\
\hline
\end{tabular}

Table 7. Distribution of galling insect orders per different localities in the Neotropical region.

Tabela 7. Distribuição das ordens de insetos galhadores por diferentes localidades na Região Neotropical.

\begin{tabular}{lcccccccc}
\hline Locality & $\begin{array}{c}\text { Dipt Cecid } \\
(\boldsymbol{\%})\end{array}$ & $\begin{array}{c}\text { Other Dipt } \\
(\boldsymbol{\%})\end{array}$ & $\begin{array}{c}\text { Lepid } \\
(\boldsymbol{\%})\end{array}$ & $\begin{array}{c}\text { Coleo } \\
(\boldsymbol{\%})\end{array}$ & $\begin{array}{c}\text { Hemip } \\
(\boldsymbol{\%})\end{array}$ & $\begin{array}{c}\text { Thysan } \\
(\boldsymbol{\%})\end{array}$ & $\begin{array}{c}\text { Hymen } \\
(\boldsymbol{\%})\end{array}$ & $\begin{array}{c}\text { Not det. } \\
(\boldsymbol{\%})\end{array}$ \\
\hline PN & 74.0 & - & - & - & 18.0 & 6.0 & - & 2.0 \\
PT & 77.4 & - & 3.8 & 4.6 & 5.3 & 2.3 & 6.0 & - \\
GO & 58.0 & 6.0 & - & - & - & - & 3.0 & 2.0 \\
SRPQ & 57.0 & - & - & - & - & 2.0 & -3 \\
BE & 86.5 & - & 7.0 & 3.2 & 1.9 & 1.3 & - \\
\hline
\end{tabular}

AC-Arraial do Cabo (Rio de Janeiro, Brazil); BE-Bertioga (São Paulo, Brazil); CP-Serra do Cipó (Minas Gerais, Brazil); EP -Espinhaço (Brazil); FB-Fazenda Bulcão (Minas Gerais, Brazil); GO-Goiania (Goiás, Brazil); GR-Grumari (Rio de Janeiro, Brazil); JU-Jurubatiba (Rio de Janeiro, Brazil); MC-Maricá and Carapebus (Rio de Janeiro, Brazil); P; PEPCV-Parque Estadual Paulo César Vinha (Espírito Santo, Brazil); PN-Panama; PT-Pantanal (Mato Grosso do Sul, Brazil); RBPS-Reserva Biológica da Praia do Sul (Rio de Janeiro, Brazil); SRPQ-Santa Rita do Passa Quatro (São Paulo, Brazil); SSJ-Serra de São José (Minas Gerais, Brazil). Cecid-Cecidomyiidae, Coleo-Coleoptera, Dipt-Diptera, Hemip-Hemiptera, Hymen-Hymenoptera, Lepid-Lepidoptera, not det.-no determined.

AC-Arraial do Cabo (Rio de Janeiro, Brasil); BE-Bertioga (São Paulo, Brasil); CP-Serra do Cipó (Minas Gerais, Brasil); EP-Espinhaço (Brasil); FB-Fazenda Bulcão (Minas Gerais, Brasil); GO-Goiânia (Goiás, Brasil); GR-Grumari (Rio de Janeiro, Brasil); JU-Jurubatiba (Rio de Janeiro, Brasil); MC-Maricá e Carapebus (Rio de Janeiro, Brasil); PEPCV-Parque Estadual Paulo César Vinha (Espírito Santo, Brasil); PN-Panamá; PT-Pantanal (Mato Grosso do Sul, Brasil); RBPS-Reserva Biológica da Praia do Sul (Rio de Janeiro, Brasil); SRPQ-Santa Rita do Passa Quatro (São Paulo, Brasil); SSJ-Serra de São José (Minas Gerais, Brasil). Cecid-Cecidomyiidae, Coleo-Coleoptera, Dipt-Diptera, Hemip-Hemiptera, Hymen-Hymenoptera, Lepid-Lepidoptera, not det.-não determinado. 
Table 7. Continued...

\begin{tabular}{|c|c|c|c|c|c|c|c|c|}
\hline Locality & $\begin{array}{c}\text { Dipt Cecid } \\
(\%)\end{array}$ & $\begin{array}{c}\text { Other Dipt } \\
(\%)\end{array}$ & $\begin{array}{c}\text { Lepid } \\
(\%)\end{array}$ & $\begin{array}{c}\text { Coleo } \\
(\%)\end{array}$ & $\begin{array}{c}\text { Hemip } \\
(\%)\end{array}$ & $\begin{array}{c}\text { Thysan } \\
(\%)\end{array}$ & $\begin{array}{c}\text { Hymen } \\
(\%)\end{array}$ & $\begin{array}{c}\text { Not det. } \\
(\%)\end{array}$ \\
\hline RBPS & 75.3 & - & 2.7 & - & 11.0 & - & - & 11.0 \\
\hline $\mathrm{MC}$ & 82.0 & - & 5.0 & 2.0 & 7.0 & $<1.0$ & 4.0 & - \\
\hline GR & 90.8 & - & 2.3 & 2.3 & 2.3 & - & - & - \\
\hline $\mathrm{AC}$ & 75.0 & - & 4.8 & - & 9.7 & 2.4 & - & 7.5 \\
\hline PEPCV & 49.0 & - & - & - & 7.0 & - & - & 44.0 \\
\hline FB & 93.0 & - & - & - & 3.5 & - & 3.5 & - \\
\hline SSJ & 74.0 & 2.8 & 7.2 & 2.8 & - & 1.4 & 2.8 & 8.4 \\
\hline $\mathrm{CP}$ & 77.0 & - & 4.0 & 1.0 & 6.0 & - & 4.0 & - \\
\hline EP & 85.0 & - & 4.0 & - & 3.0 & - & - & - \\
\hline
\end{tabular}

AC-Arraial do Cabo (Rio de Janeiro, Brazil); BE-Bertioga (São Paulo, Brazil); CP-Serra do Cipó (Minas Gerais, Brazil); EP -Espinhaço (Brazil); FB-Fazenda Bulcão (Minas Gerais, Brazil); GO-Goiania (Goiás, Brazil); GR-Grumari (Rio de Janeiro, Brazil); JU-Jurubatiba (Rio de Janeiro, Brazil); MC-Maricá and Carapebus (Rio de Janeiro, Brazil); P; PEPCV-Parque Estadual Paulo César Vinha (Espírito Santo, Brazil); PN-Panama; PT-Pantanal (Mato Grosso do Sul, Brazil); RBPS-Reserva Biológica da Praia do Sul (Rio de Janeiro, Brazil); SRPQ-Santa Rita do Passa Quatro (São Paulo, Brazil); SSJ-Serra de São José (Minas Gerais, Brazil). Cecid-Cecidomyiidae, Coleo-Coleoptera, Dipt-Diptera, Hemip-Hemiptera, Hymen-Hymenoptera, Lepid-Lepidoptera, not det.-no determined.

AC-Arraial do Cabo (Rio de Janeiro, Brasil); BE-Bertioga (São Paulo, Brasil); CP-Serra do Cipó (Minas Gerais, Brasil); EP-Espinhaço (Brasil); FB-Fazenda Bulcão (Minas Gerais, Brasil); GO-Goiânia (Goiás, Brasil); GR-Grumari (Rio de Janeiro, Brasil); JU-Jurubatiba (Rio de Janeiro, Brasil); MC-Maricá e Carapebus (Rio de Janeiro, Brasil); PEPCV-Parque Estadual Paulo César Vinha (Espírito Santo, Brasil); PN-Panamá; PT-Pantanal (Mato Grosso do Sul, Brasil); RBPS-Reserva Biológica da Praia do Sul (Rio de Janeiro, Brasil); SRPQ-Santa Rita do Passa Quatro (São Paulo, Brasil); SSJ-Serra de São José (Minas Gerais, Brasil). Cecid-Cecidomyiidae, Coleo-Coleoptera, Dipt-Diptera, Hemip-Hemiptera, Hymen-Hymenoptera, Lepid-Lepidoptera, not det.-não determinado.

Table 8. Habits of the arthropod fauna associated with insect galls in different Brazilian localities.

Tabela 8. Hábitos da fauna de artrópodes associada com galhas de insetos em diferentes localidades brasileiras.

\begin{tabular}{ccccc}
\hline Locality & Inquilines (\%) & Predators (\%) & Sucessors (\%) & Parasitoids (\%) \\
\hline GO & No data & No data & No data & 38 \\
BE & 20.0 & 2.0 & 4.7 & 24 \\
MC & 4.6 & 3.7 & No data & 56 \\
PEPCV & 10 & 2.6 & 7.9 & 31.0 \\
SSJ & 8.0 & 1.4 & No data & 33.5 \\
\hline
\end{tabular}

BE-Bertioga (São Paulo, Brazil); GO-Goiania (Goiás, Brazil); MC-Maricá and Carapebus (Rio de Janeiro, Brazil); PEPCV-Parque Estadual Paulo César Vinha (Espírito Santo, Brazil); SSJ-Serra de São José (Minas Gerais, Brazil).

BE-Bertioga (São Paulo, Brasil); GO-Goiânia (Goiás, Brasil); MC-Maricá e Carapebus (Rio de Janeiro, Brasil); PEPCV-Parque Estadual Paulo César Vinha (Espírito Santo, Brasil); SSJ-Serra de São José (Minas Gerais, Brasil).

Table 9. Arthropod fauna associated with insect galls in different Brazilian localities.

Tabela 9. Fauna de artrópodes associada com galhas de insetos em diferentes localidades brasileiras.

\begin{tabular}{|c|c|c|c|c|c|}
\hline Arthropod fauna & GO & BE & MC & PEPCV & SSJ \\
\hline \multicolumn{6}{|l|}{ Inquilines } \\
\hline Coleoptera & - & $\mathrm{x}$ & $\mathrm{x}$ & $\mathrm{x}$ & $\mathrm{x}$ \\
\hline Diptera & - & $\mathrm{x}$ & $\mathrm{x}$ & - & $\mathrm{x}$ \\
\hline Sciaridae & - & $\mathrm{x}$ & $\mathrm{x}$ & - & $\mathrm{x}$ \\
\hline Cecidomyiidae & - & $\mathrm{x}$ & $\mathrm{x}$ & - & $\mathrm{x}$ \\
\hline Camptoneuromyia sp. & - & - & $\mathrm{x}$ & - & - \\
\hline Clinodiplosis spp. & - & $\mathrm{x}$ & $\mathrm{x}$ & - & - \\
\hline Contarinia sp. & - & $\mathrm{x}$ & $\mathrm{x}$ & - & - \\
\hline Resseliella spp. & - & $\mathrm{x}$ & $\mathrm{x}$ & - & - \\
\hline Trotteria spp. & - & $\mathrm{x}$ & $\mathrm{x}$ & - & - \\
\hline Muscomorpha & - & - & - & - & $\mathrm{x}$ \\
\hline Hemiptera & - & $\mathrm{x}$ & - & - & $\mathrm{x}$ \\
\hline Hymenoptera & - & - & $\mathrm{x}$ & - & - \\
\hline Lepidoptera & - & $\mathrm{x}$ & $\mathrm{x}$ & $\mathrm{x}$ & $\mathrm{x}$ \\
\hline Thysanoptera & - & $\mathrm{x}$ & - & $\mathrm{x}$ & - \\
\hline \multicolumn{6}{|l|}{ Predators } \\
\hline Pseudoscorpiones & - & - & $\mathrm{x}$ & - & - \\
\hline Diptera & - & $\mathrm{x}$ & $\mathrm{x}$ & - & $\mathrm{x}$ \\
\hline $\begin{array}{l}\text { Cecidomyiidae } \\
\text { (Lestodiplosis sp.) }\end{array}$ & - & $\mathrm{x}$ & $\mathrm{x}$ & - & $\mathrm{x}$ \\
\hline Chloropidae & - & - & - & - & $\mathrm{x}$ \\
\hline Hymenoptera (Formicidae) & - & $\mathrm{x}$ & $\mathrm{x}$ & $\mathrm{x}$ & - \\
\hline
\end{tabular}


Table 9. Continued...

\begin{tabular}{|c|c|c|c|c|c|}
\hline Arthropod fauna & GO & $\mathbf{B E}$ & MC & PEPCV & SSJ \\
\hline \multicolumn{6}{|l|}{ Sucessors } \\
\hline Collembola & - & $\mathrm{x}$ & - & - & - \\
\hline Mites & - & - & - & $\mathrm{x}$ & - \\
\hline Psocoptera & - & $\mathrm{x}$ & - & $\mathrm{x}$ & - \\
\hline Thysanoptera & - & $\mathrm{X}$ & - & - & - \\
\hline Parasitoids (Hymenoptera) & $\mathrm{X}$ & $\mathrm{X}$ & $\mathrm{X}$ & $\mathrm{X}$ & $\mathrm{X}$ \\
\hline
\end{tabular}

\section{Acknownledgements}

I am grateful to Mineração Rio do Norte (Porto de Trombetas, Pará) for logistic and financial support , CNPq (Conselho Nacional de Desenvolvimento Científico e Tecnológico), and Antonio Clovis Britto de Araujo (Museu Nacional) for galls photographies.

\section{References}

COELHO, M.S., ALMADA, E.D., FERNANDES, G.W., CARNEIRO, M.A.A., SANTOS, R.M. \& SANCHEZ-AZOFEIFA, A. 2009. Gall inducing arthropods from a seasonally dry tropical Forest in Serra do Cipó, Brazil. Revta. Bras. Ent. 53(3):404-414. http://dx.doi.org/10.1590/ S0085-56262009000300015

DALY, D.C. 1989. The genus Tetragastris and the forests of eastern Brazil. Brittonia 41:17-27. http://dx.doi.org/10.2307/2807583

ESPÍRITO-SANTO, M., FERNANDES, G.W., ALLAIN, L.R. \& REIS, T.R.F. 1999. Tannins in Baccharis dracunculifolia (Asteraceae): effects of seasonality, water availability and plant sex. Acta Bot. Bras. 13:167-174.

FELT, E.P. 1908. Appendix D. N.Y.State Mus. Bull. 124:26-422.

FELT, E.P. 1911a. Two new gall midges (Dipt.). Entomol. News 22:109-111.

FELT, E.P. 1911b. Four new gall midges (Dipt.). Entomol. News 22:301-305.

FELT, E.P. 1915. New South America gall midges. Psyche 22:152-157.

FELT, E.P. 1917. New gall midges. J N.Y.Entomol.Soc. 25:193-196.

FELT, E.P. 1921. Three new subtropical gall midges (Itonididae, Dipt.). Entomol. News 32:141-143.

FERNANDES, G.W. \& NEGREIROS, D. 2006. A comunidade de insetos galhadores da RPPN Fazenda Bulcão, Aimorés, Minas Gerais, Brasil. Lundiana 7(2):111-120. http://dx.doi.org/10.1590/S010181751988000100002

FERNANDES, G.W., NETO, E.T. \& MARTINS, R.P. 1988. Ocorrência e caracterização de galhas entomógenas na vegetação do campus Pampulha da Universidade Federal de Minas Gerais. Rev. Bras. Zool. 5:11-29. http:// dx.doi.org/10.1590/S1676-06032010000100016

FERNANDES, S.P.C. \& MAIA, V. C. 2010. Gall midges (Diptera, Cecidomyiidae) associated with Aldina heterophylla Spr. ex. Benth. (Fabaceae) from Brazil. Biota Neotrop. 10(1):161-166.

GAGNÉ, R.J. 1969. A new genus and two new species of Cecidomyiidae associated with Pariana species (Graminae) in South America (Diptera). Proc.Entomol. Soc. Wash. 71:108-111.

GAGNÉ, R.J. 1977. The Cecidomyiidae associated with Chromolaena odorata (L.) K. and R (Compositae) in South America. Brenesia 12-13:113-31.

GAGNÉ, R.J. 1989. The Plant-feeding gall midges of North America. Cornell University Press, Ithaca, 356p.

GAGNÉ, R.J. 1994. The Gall Midges of the Neotropical Region. Cornell University Press, Ithaca, 352p.

GAGNÉ, R.J. \& Hibbard, K.L. 1996. A new species of gall midge (Diptera, Cecidomyiidae) from subterraneous stem galls of Licania michauxii (Chrysobalanaceae) in Florida. Fla. Entomol. 79(3):428-434. http:// dx.doi.org/10.2307/3495593

HOUARD, C. 1924. Les Collections cécidologiques du Laboratoire d'Entomologie du Muséum d'Histoire naturelle de Paris: Galles de Guyanne française (Premier Mémoire). Marcellia 21: 97-128.
HOUARD, C. 1933. Les Zoocécidies des Plantes de L'Amérique Du Sud et de L'Amérique Centrale. Hermann et Cie, 519p.

JULIÃO, G.R. 2007. Riqueza e abundância de insetos galhadores associados ao dossel de florestas de terra firme, várzea e igapó da Amazônia Central. Tese de Doutorado, Universidade Federal do Amazonas, Manaus, 144p.

KIEFFER, J.J. 1895. Bull. Bimens. Soc. Entomol. France 63(1894):cclxxx.

KIEFFER, J.J. 1913. Diptera. Fam. Cecidomyiidae. Fasc. 152, 346 pp. 15 pls. In Wytsman P. ed. Genera Insectorum, Bruxelles.

LARA, D.P., OLIVEIRA, L.A., AZEVEDO, I.F.P., XAVIER, M.F., SILVEIRA, F.A.O., CARNEIRO, M.A.A. \& FERNANDES, G.W. 2008. Relationships between host plant architecture and gall abundance and survival. Rev. Bras. Entomol. 52(1):78-81. http://dx.doi.org/10.1590/ S0085-56262008000100014

LAWTON, J.H. \& SHRÖDER, D. 1977. Effects of plant type, size of geographical range and taxonomic isolation on number of insects associated with British plants. Nature 265:137-140. http://dx.doi. org/10.1038/265137a0

MAIA, V.C. 2001. The gall midges (Diptera, Cecidomyiidae) from three restingas of Rio de Janeiro State, Brazil. Revta Bras. Zool. 18(2):583-629. http://dx.doi.org/10.1590/S0101-81752001000200028

MAIA, V.C., Azevedo, A.P. de \& Couri, M.S. 2002. New contribution to the knowlegde of the gall midges (Diptera, Cecidomyiidae) from the restinga of Barra de Maricá (Rio de Janeiro, Brazil). Studia Dipt. 447-452.

MAIA, V.C. \& Fernandes, G.W. 2004. Insect galls from Serra de São José (Tiradentes, MG, Brazil. Braz. J. Biol. 64(3a):423-445. http://dx.doi. org/10.1590/S1519-69842004000300007

MAIA, V.C. \& Fernandes, G.W. 2006. A new genus and species of gall midge (Diptera, Cecidomyiidae) associated with Parkia pendula (Fabaceae, Mimosoidea). Rev. Bras. Entomol. 50(1):1-5.

MAIA, V.C., Magenta, M.A. \& Martins, S.E. 2008. Ocorrência e caracterização de galhas de insetos em áreas de restinga de Bertioga (São Paulo, Brasil). Biota Neotrop. 8(1):167-197. http://dx.doi.org/10.1590/ S1676-06032008000100020

MAIA, V.C. \& Vásquez, J. 2006. A new species of gall midge (Diptera: Cecidomyiidae) associated with Theobroma bicolor (Sterculiaceae) from Peru. Arq. Mus. Nac. 64(2):125-129.

MANI, M.S. 1964. Ecology of Plant Galls. Dr. W. Junk, The Hague, 434p.

MARQUES, D.D., SARTORI, R.A., LEMOS, T.L.G., MACHADO, L.L., SOUZA, J.S.N. \& MONTE, F.J.Q. 2010. Chemical composition of the essential oils from two subspecies of Protium heptaphyllum. Acta Amazon. 40(1):227-230. http://dx.doi.org/10.1590/S004459672010000100029

MATA, M.F. \& FELIX, L.P. 2007. Flora da Paraíba, Brasil: Inga Mill. (Leguminosae - Mimosoideae). Rev. Bras. Biociênc. 5(2):135-137.

MÖHN, E. 1959. Gallmücken (Diptera, Itonididae) aus El Salvador. 1. Teil. Senckenbergiana Biol. 40:297-368.

MÖHN, E. 1960. Gallmücken (Diptera, Itonididae) aus El Salvador. 2. Teil. Senckenbergiana Biol 41:197-240.

MÖHN, E. 1962. Sudien über neotropische Gallmücken(Diptera, Itonididae). 1. Teil. Broteria 31:211-239.

MÖHN, E. 1964. Gallmücken (Diptera, Itonididae) aus El Salvador. 6.Teil, Lasiopteridi. Dtsch. Entomol. Z. 11:47-143.

MÖHN, E. 1975. Gallmücken (Diptera, Itonididae) aus El Salvador. 8.Teil, Lasiopteridi. Sttutgarter Beitr. Naturk. (A)276:1-101. 
MOLLIARD, M. 1903. La galle du Cecidomyia cattleya n. sp. Marcellia 1(1902):165-170.

MONTEIRO, R.F., FERRAZ, F.F.F., MAIA, V.C. \& AZEVEDO, M.A. 1993 Galhas entomógenas em restingas: uma abordagem preliminar. An. III Simp. Ecossist. Costa Brasileira, ACIESP: 210-221.

NIEVES-ALDREY, J.L., IBÁNEZ, A. \& MEDIANERO, E. 2008. Richness and composition of gall-inducing arthropods at Coiba National Park, Panama. Rev. Biol.Trop. 56(3):1269-1286.

ODA, R.A.M. 2006. Distribuição espacial de insetos fitófagos, com ênfase em galhadores, em três diferentes regiões do Brasil. Tese de Doutorado, Universidade Federal do Rio de Janeiro, Rio de Janeiro, 105p.

OLIVEIRA, D.C., DRUMMOND, M.M., MOREIRA, A.S.F.P., SOARES, G.L.G. \& ISAIAS, R.M.S. 2008. Potencialidades morfogênicas de Copaifera langsdorffi Desf. (Fabaceae): super-hospedeira de herbívoros galhadores. Rev. Biol. Neotrop. 5(1):31-39.

RÜBSAAMEN, E.H. 1895. Cecidomyidenstudien. Entomol. Nach. 21:177194.

RÜBSAAMEN, E.H. 1905. Beiträge zur Kenntnis aussereuropäischer Zoocecidien. II. Beitrag: Gallen aus Brasilien und Peru. Marcellia 4:65-85.

RÜBSAAMEN, E.H. 1907. Beiträge zur Kenntnis aussereuropäischer Zoocecidien. III. Beitrag: Gallen aus Brasilien und Peru. Marcellia 6:110-173.

RÜBSAAMEN, E.H. 1908. Beiträge zur Kenntnis aussereuropäischer Zoocecidien. III. Beitrag (cont.): Gallen aus Brasilien und Peru. Marcellia 7:15-79.

RÜBSAAMEN, E.H. 1916a. Beitrag zur Kenntnis aussereuropäischer Gallmücken. Sitzßer Ges. natf. Freunde Berlin 1915:431-481.
RÜBSAAMEN, E.H. 1916b. Cecidomyidenstudien V. Revision der deutschen Asphondylarien. Sitzßer Ges. natf. Freunde Berlin 1916:1-12.

SANTOS, B.B., FERREIRA, H.D. \& ARAÚJO, W.S. 2010. Ocorrência e caracterização de galhas entomógenas em uma área de floresta estacional semidecídua em Goiânia, Goiás, Brasil. Acta Bot. Bras. 24(1):243-249. http://dx.doi.org/10.1590/S0102-33062010000100026

SILVA, A.L.G. \& PINHEIRO, M.C.B. 2007. Biologia floral e da polinização de quatro espécies de Eugenia L. (Myrtaceae) Acta bot. bras. 21(1):235-247.

SILVESTRI, F. 1901. Descrizione di nuovi termitofili e rellazioni di essi com gli ospiti. Boll. Mus.Zool. Anat. Comp. Univers. Torino 16(395):1-6.

TAVARES, J.S. 1917. As cecídias do Brazil que se criam nas plantas da família das Melastomataceae. Brotéria, Zool. 15:18-49.

TAVARES, J.S. 1918. Cecidologia brazileira. Cecídias que se criam nas plantas das famílias das Verbenaceae, Euphorbiaceae, Malvaceae, Anacardiaceae, Labiatae, Rosaceae, Anonaceae, Ampelidaceae, Bignoniaceae, Aristolochiaceae e Solanaceae. Brotéria, Zool. 16:21-68.

TAVARES, J.S. 1920. Cecidologia brazileira. Cecídias que se criam em plantas das famílias das Leguminosae, Sapotaceae, Lauraceae, Myrtaceae, Punicaceae, Aurantiaceae, Malpighiaceae, Sapindaceae, Umbelliferae, Loranthaceae, Apocynaceae, Urticaceae, Salicaceae e Gramineae. Brotéria, Zool. 18:82-125.

TAVARES, J.S. 1922. Cecidologia brazileira. As restantes famílias. Brotéria, Zool. 20:5-48.

TAVARES, J.S. 1925. Nova contribuição para o conhecimento da cecidologia brazileira. Brotéria, Zool. 22:5-55

WÜNSCHE, A. 1979. Gallenerzeugende Insekten Nordkolumbiens, speziell Asphondyliidi und Lasioperidi (Diptera, Cecidomyiidae) aus dem Küstenbereich um Santa Marta. Waiblingen, West Germany, 238p. 
